\title{
Are Serum Levels of Inflammatory Markers Associated With the Severity of Symptoms of Bipolar Disorder?
}

\author{
Xiuhua Wu \\ Third Affiliated Hospital of Sun Yat-sen University \\ Zhongcheng Chen \\ Third Affiliated Hospital of Sun Yat-sen University \\ Yingtao Liao \\ Third Affiliated Hospital of Sun Yat-sen University \\ Zhihua Yang \\ Third Affiliated Hospital of Sun Yat-sen University \\ Xiaolin Liang \\ Third Affiliated Hospital of Sun Yat-sen University \\ Nianhong Guan \\ Third Affiliated Hospital of Sun Yat-sen University \\ Zhaoyu Gan ( $\nabla$ ganzhy@mail.sysu.edu.cn ) \\ Third Affiliated Hospital of Sun Yat-sen University
}

Research Article

Keywords: bipolar disorder, symptomatic severity, inflammatory markers, stage

Posted Date: February 7th, 2022

DOI: https://doi.org/10.21203/rs.3.rs-1250412/v1

License: (c) (i) This work is licensed under a Creative Commons Attribution 4.0 International License. Read Full License 


\section{Abstract}

Background: To explore the relationship between serum levels of inflammatory markers and symptomatic severity of bipolar disorder (BD).

Material and Methods: A cross-sectional study was conducted on 126 BD patients with current depressive episode (BDD), 102 BD patient with current mixed or (hypo)manic episode (BDM) and 94 healthy controls (HC). Fasting serum levels of CRP, leptin (LEP), adiponectin (ADP), visfatin (VIS), TNF-a, IL-2, IL-6, IL-10, IL-17), and monocyte chemoattractant protein-1 (MCP-1) were measured with enzyme-linked immunosorbent assay (ELISA). Symptomatic severity of BD was assessed with HAMD-17 and YMRS. Generalized linear model was used to determine the association between the serum levels of inflammatory markers and symptomatic severity of BD.

Results: The serum levels of IL-6, IL-10 and IL-17, and the IL-6/IL-10 ratio were significantly lower in mild BDD than in HC. In moderate BDD, the serum levels of MCP, IL-6 and IL-17 were significantly lower than in HC. In severe BDD, the serum level of ADP, MCP-1, IL-10 and IL-17and the IL-17/IL-10 ratio were significantly lower than in $\mathrm{HC}$. The serum levels of TNF- $a$ and the IL-6/IL-10 ratio were significantly higher in mild BDM than in HC. In moderate BDM, the serum level of VIS, IL-2, and IL-17 were significantly higher than in HC, but the IL-6/IL-10 ratio was significantly lower than in control. In severe BDM, the serum levels of IL-6 and IL-17 and the ratios of IL-6/IL-10 and IL-17/IL-10 were significantly lower than in HC, but the neutrophil/lymphocyte ratio was significantly higher than in HC.

Conclusions: In BDD, immune-inhibition is persistently predominant, while in BDM, immune system is first activated then inhibited with the worsening of the symptoms. The dynamic change of serum inflammatory markers might help stage BD and guide the future immune treatment of BD.

\section{Background}

Since Kraepelin first noted that bipolar disorder (BD) had an accelerating and progressive course in 1921[1], several clinical staging systems have been put forward to stage BD with peripheral biomarkers.[2], and one of the staging systems is immune system [3]. Over the past decade, growing and converging evidence has demonstrated that immune dysfunction is involved in the pathophysiology of BD [4]. Therefore, BD is viewed as a multi-system inflammatory disease[5] and anti-inflammatory agents are thought to be a potential treatment option of BD [6]. However, inflammatory markers, as biomarkers to stage BD, have been seldom studied. As far as we know, only one systematic review [3] has ever summarize the available literature of studies about the clinical variables related with staging BD patients. According to this review, the most frequently evaluated clinical variables related to BD staging were onset age, duration of illness course, number of affective episodes and cognitive function, but few studies have ever used symptomatic severity as a clinical variable to stage BD and associate it with peripheral inflammatory markers. This paucity of data is further problematic since symptoms are not only the target of treatment but also reflect the severity of illness, and the latter is an important clinical variable associated with the stage of BD[7].

Elevated pro-inflammatory cytokines had been reported to be associated with greater symptom burden in BD [8, 9]. However, this way that treats the relationship between serum levels of inflammatory markers and symptomatic severity as a linear one might simplify their association. Supposing BD was a multi-system inflammatory disease as someone claimed [5], the serum levels of inflammatory markers would first increase with the worsening of symptoms and then decrease because of exhaustion with the evolvement of illness like other inflammatory diseases [10]. Additionally, peripheral inflammatory biomarkers are variable and heterogeneous. Lots of factors might affect the serum level of inflammatory biomarkers, including gender[11], age[12], marital status[13], body mass index (BMI) [14], psychoactive substance abuse[11], atypical features[15], duration of illness[16], pharmaceutical treatment[17], and so on. Finally, among the peripheral inflammatory biomarkers, some are associated with mood states (state markers) and some are related to specific features of the long-term course of illness (trait markers)[18]. This further complicates the relationship between serum levels of inflammatory biomarkers and the stage of $\mathrm{BD}$ and poses a big challenge in studies of this area.

In this study, we are going to assess the relationship between serum levels of inflammatory cytokines and symptomatic severity of bipolar disorder in a large, drug-naive BD population, aiming to explore the possibility of serum levels of inflammatory cytokines as biomarkers to stage BD and guide the future immune treatment of $\mathrm{BD}$ if possible.

\section{Materials And Methods}

\section{Subjects}

The sample consisted of 228 BD patients and 94 healthy controls. The cases came from the inpatients or outpatients who sought medical help in the psychiatric department of the Third Affiliated Hospital of Sun Yat-sen University between August 8, 2012 and January 6, 2018. The healthy controls were volunteers recruited from the local community during the same period. All the participants were Han Chinese, aged between 16 and 65 , had no current active physical illness associated with inflammatory response confirmed by reviewing their previous medical records and routine clinical examination, had no history of psychoactive substance abuse in the past six months, and provided written informed consent. Participants who aged under 18 were required to provide written informed consent from their guardians. The cases had to meet the following criteria: a) fulfill the diagnostic criteria of bipolar disorder of any kind according to the Diagnostic and Statistical Manual of Mental Disorders, Fourth Edition, Text Revision (DSM- -TR);b)had not received any psychopharmaceutical treatment within 3 months prior to recruitment; c) had no comorbid organic mental disorder. The healthy controls were screened for mental disorders using the Chinese version of the Structured Clinical Interview for the Diagnostic and Statistical Manual of Mental Disorders, Fourth Edition, Text Revision (DSM-IV-TR) Axis 1 Disorders (SCID-I), none patient version, and those with a current or history of major psychiatric disorders, dementia, mental retardation, would be excluded. In addition, participants who were pregnant or postpartum, under steroid or non-steroid anti-inflammatory drugs or other immune-inhibitors treatment were also excluded. This study was reviewed and approved by the Clinical Research Ethics Committee of the Third Affiliated Hospital of Sun Yat-sen University. 


\section{Measurement \\ Diagnosis of BD}

The diagnosis of BD was based on the structural interview with the Chinese version of the Structured Clinical Interview for the Diagnostic and Statistical Manual of Mental Disorders, Fourth Edition, Text Revision (DSM-IV-TR) Axis 1 Disorders (SCID-I), patient version.

\section{Assessment of the symptomatic severity of BD}

The symptomatic severity of BD was evaluated with the 17-item Hamilton Depression Scale (HAMD-17) [19] and the Young Mania Rating Scale (YMRS)[20].

\section{Biochemical measurement}

Ten milliliters of fasting blood were withdrawn between 7:00am and 9:00 am from each subject by venipuncture into two free-anticoagulant vacuum tubes. One tube of blood was immediately sent to the library of our hospital for routine blood test and measurement of $\mathrm{C}$ reactive protein (CRP). Routine blood test was performed in a automated blood cell counter (Hiesen Mikang co., Ltd, Kōbe, Japan). CRP were measured by immune transmission turbidimetry with a biochemical analyzer (Sichuan Mike Biological Co., Ltd and Nippon ihua co., Ltd, Tokyo, Japan). The other tube of blood was immediately centrifuged at $3000 \mathrm{~g}$ for $5 \mathrm{~min}$, and the serum was kept frozen at $-80^{\circ} \mathrm{C}$ until assayed. The concentrations of leptin (LEP) adiponectin (ADP) visfatin (VIS), tumor necrosis factor-a (TNF-a), interleukin-2 (IL-2), interleukin-6 ( IL-6), interleukin-10 (IL-10), interleukin-17 (IL-17), and monocyte chemoattractant protein-1 (MCP-1) were assessed with enzyme-linked immunosorbent assay (ELISA) using assay from Shanghai Caiyou Industrial Co. Ltd.

\section{Statistic analysis}

Participants were categorized into BD group and HC group according to the inclusion criteria, and the BD group was further divided into two subgroups-depressive group and (hypo)manic or mixed group based on their current episode. Patients with current mixed episode was classified as (hypo) manic group, considering the number of patients with current (hypo)manic episode was small in this study and (hypo)manic and mixed episode shared similar treatment strategy[21]. The scores of HAMD-17 and YMRS were treated as a categorized variables, for HAMD-17: 0 (no depression): $<7 ; 1$ mild depression : $\geq 7$ and 17 2 (moderate depression): $\geq 17$ and $<24 ; 3$ (severe depression): $\geq 24$ for YMRS: 0 (no mania): $<6 ; 1$ (mild mania): $\geq 6$ and $<132$ moderate mania $: \geq 13$ and $<19 ; 3$ (server mania): $\geq 19$. For normally distributed data, independent-sample t-test was used to test the difference between groups, while for non-normally distributed variable, comparison between groups was analyzed with Wilcoxon rank sum test. Difference in categorical parameters between groups was tested using Chi-square test. Generalized linear model was built to assess the impact of severity of symptoms on the serum levels of inflammatory markers. Odds ratios (OR) and $95 \%$ confidence intervals $(95 \% \mathrm{Cl}$ ) were used to quantify the strength of associations. The Bonferroni adjusted significance test was used for multiple comparisons. All data were analyzed using commercial statistical package SPSS 24.0 (SPSS, Inc., Chicago, IL).

\section{Results}

\section{Demographic and clinical characteristics of participants}

As seen in Table1, 126 BD patients with current depressive episode (BDD), 102 BD patients with current (hypo)manic or mixed episode (BDM) and 94 healthy controls $(\mathrm{HC})$ were recruited in this study. The level of education and the proportion of female participants among BDD were significantly lower than those among $\mathrm{HC}(\mathrm{P}<0.001)$, but no significant difference was found in age, marital status, and BMI between BDD and HC. Compared to HC, BDM was younger $(P<0.001)$ and had a lower level of education $(P<0.001)$, but did not significantly differ in gender proportion, marital status and $B M I$. 
Table 1

Demographic and clinical characteristics of participants

\begin{tabular}{|c|c|c|c|c|c|}
\hline & \multicolumn{2}{|c|}{ Bipolar disorder } & \multirow{3}{*}{$\begin{array}{l}\text { Control } \\
\mathrm{N}=94\end{array}$} & \multirow[t]{3}{*}{$P^{1}$} & \multirow[t]{3}{*}{$P^{2}$} \\
\hline & Depressive & (hypo)manic & & & \\
\hline & $N=126$ & $\mathrm{~N}=102$ & & & \\
\hline Age (mean $\pm S D$ )(year) & $27.8 \pm 10.7$ & $25.4 \pm 8.1$ & $29.6 \pm 11.0$ & 0.241 & 0.002 \\
\hline Female n(\%) & $65(51.6)$ & $66(64.7)$ & $68(72.3)$ & 0.002 & 0.284 \\
\hline Education (mean $\pm S D$ ) (year) & $13.0 \pm 3.4$ & $12.8 \pm 3.0$ & $16.2 \pm 3,5$ & $<0.001$ & $<0.001$ \\
\hline Married n (\%) & $42(33.3)$ & $29(28.4)$ & $28(29.8)$ & 0.661 & 0.876 \\
\hline $\mathrm{BMI}($ mean $\pm S D)$ & $21.7 \pm 2.9$ & $21.5 \pm 3.6$ & $21.5 \pm 3.8$ & 0.674 & 0.997 \\
\hline \multicolumn{6}{|l|}{ Severity of symptoms } \\
\hline 1 (mild) & 17 & 35 & NA & & \\
\hline 2( moderate) & 35 & 33 & NA & & \\
\hline 3(severe) & 74 & 33 & NA & & \\
\hline \multicolumn{6}{|l|}{ Note: } \\
\hline \multicolumn{6}{|c|}{ 1. Bipolar depression compared with Control; } \\
\hline \multicolumn{6}{|c|}{ 2. Bipolar disorder with current (hypo)manic or mixed episode compared with Control. } \\
\hline \multicolumn{6}{|l|}{ 3. BMI: body mass index } \\
\hline 4. Bold font denotes $p<0.05$ & & & & & \\
\hline
\end{tabular}

\section{The association between severity of depressive symptoms and serum level of inflammatory markers}

Table 2 demonstrated that compared to $\mathrm{HC}$, most of the observed inflammatory markers, including pro-inflammatory and anti-inflammatory markers, showed a trend of decrease in BDD of any degree. In mild BDD, the serum level of IL- $6\left(P=0.003, O R=e^{-1.087}=0.337,95 \% C l=e^{-1.807}-e^{-0.366}=0.164-0.693\right)$, IL-10 $(P=0.002$, $\left.\mathrm{OR}=\mathrm{e}^{-1.069}=0.343,95 \% \mathrm{Cl}=\mathrm{e}^{-1.746}-\mathrm{e}^{-0.393}=0.174-0.675\right)$ and IL-17( $\left.\mathrm{P}<0.001, \mathrm{OR}=\mathrm{e}^{-1.203}=0.300,95 \% \mathrm{Cl}=\mathrm{e}^{-1.81}-\mathrm{e}^{-0.595}=0.164-0.551\right)$ were significantly lower than those in $\mathrm{HC}$, the IL-6/IL-10 ratio was significantly lower $\left(P=0.008, O R=e^{-0.892}=0.410,95 \% C l=e^{-1.544}-e^{-0.23}=0.311-0.990\right)$ in mild $B D D$ than in health control, but after Bonferroni correction, the difference did not reach significance. In moderate $B D D$, the serum level of $M C P\left(P=0.002, O R=e^{-733}=0.480,95 \% C l=e^{-1.194}\right.$ $\left.\mathrm{e}^{-0.272}=0.303-0.762\right), \mathrm{IL}-6\left(\mathrm{P}<0.001, \mathrm{OR}=\mathrm{e}^{-0.928}=0.395,95 \% \mathrm{Cl}=\mathrm{e}^{-1.41}-\mathrm{e}^{-0.446}=0.244-0.640\right)$ and IL-17 $\left(\mathrm{P}<0.001, \mathrm{OR}=\mathrm{e}^{-1.035}=0.355,95 \% \mathrm{Cl}=\mathrm{e}^{-1.478}-\mathrm{e}^{-0.593}=0.228-\right.$ 0.553 ) were significantly lower than those in HC, but no significant difference was found in the ratios of IL-2/IL-10, IL-6/IL-10, IL-17/IL-10 and neutrophil/lymphocyte $(N / L)$ between $B D D$ and $H C$. In severe $B D D$, the serum level of $A D P\left(P=0.002, O R=e^{-0.81}=0.445,95 \% C l=e^{-0.915}-e^{-0.199}=0.400-0.820\right)$, $\operatorname{MCP}-1\left(P<0.001, O R=e^{-0.557}=0.573,95 \% C l=e^{-1.179}-e^{-0.44}=0.308-0.644\right), I L-10\left(P=0.001, O R=e^{-0.616}=0.540,95 \% C l=e^{-0.979}-e^{-0.253}=0.376-0.776\right.$, and IL$17\left(\mathrm{P}<0.001, \mathrm{OR}=\mathrm{e}^{-1.235}=0.291,95 \% \mathrm{Cl}=\mathrm{e}^{-1.607}-\mathrm{e}^{-0.863}=0.200-0.422\right)$ were significantly lower than those in $\mathrm{HC}$, and the IL-17/IL-10 ratio was aslo lower $\left(\mathrm{P}<0.001, \mathrm{OR}=\mathrm{e}^{-0.752}=0.471,95 \% \mathrm{Cl}=\mathrm{e}^{-1.091}-\mathrm{e}^{-0.412}=0.335-0.662\right)$. 
Table 2

The association between severity of depressive symptoms and serum level of inflammatory markers

\begin{tabular}{|c|c|c|c|c|c|c|c|c|c|c|c|c|c|c|}
\hline \multirow{3}{*}{$\begin{array}{l}\text { Inflammatory } \\
\text { markers }\end{array}$} & \multirow[t]{3}{*}{$\mathbf{N}$} & \multirow{3}{*}{$\begin{array}{l}\text { Goodness } \\
\text { of fit } \\
\text { (value/df) }\end{array}$} & \multirow{3}{*}{$\begin{array}{l}\text { Omnibus } \\
\text { Test } \\
(P \mathbb{X})\end{array}$} & \multicolumn{11}{|l|}{$\mathrm{BDD}^{1}$} \\
\hline & & & & \multicolumn{4}{|l|}{ mild } & \multicolumn{4}{|c|}{ moderate } & \multicolumn{3}{|l|}{ severe } \\
\hline & & & & $\beta^{2}$ & $95 \% \mathrm{Cl}$ & & $p^{3}$ & $\beta^{2}$ & $95 \% \mathrm{Cl}$ & & $P^{3}$ & $\beta^{2}$ & $95 \% \mathrm{Cl}$ & \\
\hline $\mathrm{WBC}^{4}$ & 156 & 3.312 & 0.463 & -0.409 & -1.606 & 0.789 & 0.504 & 0.037 & -0.793 & 0.868 & 0.930 & 0.400 & -0.241 & 1.042 \\
\hline $\mathrm{CRP}^{5}$ & 172 & 0.951 & 0.535 & -0.121 & -0.691 & 0.449 & 0.677 & -0.175 & -0.593 & 0.243 & 0.412 & -0.225 & -0.535 & 0.085 \\
\hline$A D P^{5}$ & 210 & 1.612 & 0.013 & -0.482 & -1.149 & 0.185 & 0.157 & -0.481 & -0.928 & -0.034 & 0.035 & -0.557 & -0.915 & -0.199 \\
\hline LEP $^{5}$ & 210 & 1.591 & 0.113 & -0.295 & -.0959 & 0.368 & 0.383 & 0.188 & -0.257 & 0.632 & 0.407 & -0.330 & -0.686 & 0.026 \\
\hline $\mathrm{VIS}^{5}$ & 210 & 1.322 & 0.512 & -0.255 & -.866 & 0.357 & 0.414 & -0.218 & -0.627 & 0.191 & 0.297 & -0.217 & -0.545 & 0.111 \\
\hline MCP- $1^{5}$ & 210 & 1.730 & $<0.001$ & -0.904 & -1.592 & -0.215 & 0.010 & -0.733 & -1.194 & -0.272 & 0.002 & -0.810 & -1.179 & -0.440 \\
\hline $\mathrm{IL}-2^{5}$ & 156 & 0.794 & 0.392 & -0.048 & -0.552 & 0.457 & 0.853 & -0.162 & -0.530 & 0.205 & 0.386 & 0.153 & -0.156 & 0.462 \\
\hline IL- $6^{5}$ & 210 & 1.914 & $<0.001$ & -1.087 & -1.807 & -0.366 & 0.003 & -0.928 & -1.410 & -0.446 & $<0.001$ & -0.544 & -0.930 & -0.158 \\
\hline IL-10 $10^{5}$ & 210 & 1.665 & 0.001 & -1.069 & -1.746 & -0.393 & 0.002 & -0.581 & -1.034 & -0.128 & 0.012 & -0.616 & -.979 & -0.253 \\
\hline $\mathrm{IL}-17^{5}$ & 156 & 1.197 & $<0.001$ & -1.203 & -1.810 & -0.595 & $<0.001$ & -1.035 & $-1,478$ & -0.593 & $<0.001$ & -1.235 & -1.607 & -0.863 \\
\hline TNF-a ${ }^{5}$ & 210 & 1.215 & 0.405 & -0.106 & -0.694 & 0.483 & 0.725 & -0.319 & -0.714 & 0.075 & 0.112 & 0.027 & -0.288 & 0.343 \\
\hline$N / L^{6}$ & 154 & 0.206 & 0.328 & -0.182 & -0.476 & 0.113 & 0.226 & 0.003 & -0.201 & 0.208 & 0.975 & 0.087 & -0.071 & 0.246 \\
\hline IL-2/IL-10 5 & 156 & 0.921 & 0.403 & 0.382 & -0.157 & 0.922 & 0.165 & -0.024 & -0.417 & 0.369 & 0.904 & 0.162 & -0.169 & 0.493 \\
\hline IL-6/IL-10 & 210 & 1.584 & 0.032 & -0.892 & -1.554 & -0.230 & 0.008 & -0.361 & -0.805 & 0.082 & 0.110 & -0.368 & -0.724 & -0.013 \\
\hline IL-17/IL-10 5 & 156 & 0.978 & 0.001 & -0.319 & -0.874 & 0.235 & 0.257 & -0.296 & -0.699 & 0.108 & 0.151 & -0.752 & -1.091 & -0.412 \\
\hline
\end{tabular}

Note:

1. Adjustment for age, gender, marital status, BMI, education, and severity of manic symptoms.

2. Taking the healthy control group as reference, if the $\beta$ is positive, the level of inflammatory marker is higher than that of healthy control group, and if the $\beta i$ negative, the level of inflammatory marker is lower than that of healthy control group

3. Bold font denotes $p<0.05 / 15=0.0033$

4. According to the scatter plot and statistical test, these data are approximately normally distributed, so the linear model is used in modeling;

5. According to the scatter plot and statistical test, these data are skewed distributed, but approximately normally distributed after log conversion, so gamma link model is chosen for modeling.

6. N/L: Granulocyte/lymphocyte ratio

\section{The association between severity of manic symptoms and serum level of inflammatory markers}

Table 3 showed a different change model of serum level of inflammatory markers across manic symptoms of varying degree. With the worsening of manic symptoms, most of the observed pro-inflammatory markers were seen to elevate first then decrease, but the anti-inflammatory marker, IL-10, decreased first then increased and finally decreased, though the change did not reach significance. In mild $B D M$, only the serum level of $T N F-a\left(P<0.001,0 R=e^{1.000}=2.718\right.$, $\left.95 \% \mathrm{Cl}=\mathrm{e}^{0.490}-\mathrm{e}^{1.511}=1.632-4.531\right)$ was significantly higher than that in $\mathrm{HC}$, but the IL-6/IL-10 ratio was significantly lower $\left(P<0.001,0 R=e^{1.141}=3.130,95 \% C l=\right.$ $\left.\mathrm{e}^{0.586}-\mathrm{e}^{1.696}=1.797-5.452\right)$ in case than in control. In moderate BDM, the serum levels of VIS $\left(P<0.001,0 R=e^{0.862}=2.368,95 \% C l=e^{0.419}-e^{1.305}=1.520-3.688\right), I L-$ $2\left(P<0.001, O R=e^{0.983}=2.672,95 \% C l=e^{0.606}-e^{1.361}=1.833-3.900\right)$, and $I L-17\left(P<0.001, O R=e^{1.375}=3.955,95 \% C l=e^{0.951}-e^{1.798}=2.588-6.038\right)$, were seen to be significantly higher than in $\mathrm{HC}$, but the IL-6/IL-10 ratio was also significantly lower $\left(P=0.003, O R=e^{-0.685}=0.504,95 \% C l=e^{-1.141}\right.$ - $\left.e^{-0230}=0.319-0.794\right)$ in the case group. However, when the manic symptoms further worsen to the severe degree, most of the observed pro-inflammatory markers decreased. Of them, IL-6 $\left(P=0.001, O R=e^{-0.813}=0.443,95 \% C l=e^{-1.281}-e^{-0.346}=0.278-0.708\right)$ and IL-17 $\left(P<0.001, O R=e^{-1.110}=0.330,95 \% C l=e^{-1.716}-e^{-0.504}=0.180-0.604\right)$ reached significance. In addition, the ratios of IL-6/IL-10 ( $\left.P<0.001, \mathrm{OR}=\mathrm{e}^{-1.011}=0.364,95 \% \mathrm{Cl}=\mathrm{e}^{-1.442}-\mathrm{e}^{-0.580}=0.236-0.560\right)$ and IL-17/IL-10 $\left(P=0.001, \mathrm{OR}=\mathrm{e}^{-0.761}=0.467\right.$, $95 \% \mathrm{Cl}=\mathrm{e}^{-1.209}-\mathrm{e}^{-0.314}=0.298-0.730$ ) were significantly lower in the case group than in the control group. Finally, the N/L ratio was found to be significantly higher in severe $\mathrm{BDM}$ than in $\mathrm{HC}\left(\mathrm{P}=0.001, \mathrm{OR}=\mathrm{e}^{0.499}=1.647,95 \% \mathrm{Cl}=\mathrm{e}^{0.212}-\mathrm{e}^{0.787}=1.236-2.198\right)$. 
Table 3

The association between severity of manic symptoms and serum level of inflammatory markers

\begin{tabular}{|c|c|c|c|c|c|c|c|c|c|c|c|c|c|c|}
\hline \multirow[t]{3}{*}{$\begin{array}{l}\text { Inflammatory } \\
\text { markers }\end{array}$} & \multirow[t]{3}{*}{$\mathbf{N}$} & \multirow{3}{*}{$\begin{array}{l}\text { Goodness } \\
\text { of fit } \\
\text { (value/df) }\end{array}$} & \multirow{3}{*}{$\begin{array}{l}\text { Omnibus } \\
\text { Test } \\
(P X)\end{array}$} & \multicolumn{11}{|l|}{$\mathrm{BDM}^{1}$} \\
\hline & & & & \multicolumn{4}{|l|}{ mild } & \multicolumn{4}{|c|}{ moderate } & \multicolumn{3}{|l|}{ severe } \\
\hline & & & & $\beta^{2}$ & $95 \% \mathrm{Cl}$ & & $p^{3}$ & $\beta^{2}$ & $95 \% \mathrm{Cl}$ & & $p^{3}$ & $\beta^{2}$ & $95 \% \mathrm{Cl}$ & \\
\hline $\mathrm{WBC}^{4}$ & $146^{1}$ & 3.807 & 0.345 & 0.462 & -0.685 & 1.609 & 0.430 & 0.761 & -0.074 & 1.595 & 0.074 & 0.228 & -0.538 & 0.994 \\
\hline $\mathrm{CRP}^{5}$ & $161^{6}$ & 1.452 & 0.065 & -0.238 & -0.800 & 0.323 & 0.406 & 0.472 & 0.074 & 0.869 & 0.020 & 0.166 & -0.199 & 0.531 \\
\hline$A D P^{5}$ & 189 & 2.196 & 0.022 & 0.604 & -0.018 & 1.227 & 0.057 & 0.401 & -0.116 & 0.918 & 0.128 & -0.319 & -0.802 & 0.164 \\
\hline LEP 5 & 190 & 1.6741 & 0.010 & 0.309 & -0.244 & 0.862 & 0.273 & 0.458 & 0.004 & 0.912 & 0.048 & -0.412 & -0.814 & 0.017 \\
\hline $\mathrm{VIS}^{5}$ & 190 & 1.584 & $<0.001$ & 0.068 & -0.472 & 0.608 & 0.805 & 0.862 & 0.419 & 1.305 & $<0.001$ & -0.281 & -0.700 & 0.138 \\
\hline MCP- $1^{5}$ & 191 & 2.029 & 0.082 & -0.355 & -0.957 & 0.246 & 0.247 & 0.023 & -0.471 & 0.517 & 0.929 & -0.584 & -1.047 & -0.121 \\
\hline IL-2 ${ }^{6}$ & 144 & 52.166 & $<0.001$ & 0.499 & 0.004 & 0.995 & 0.048 & 0.983 & 0.606 & 1.361 & $<0.001$ & 0.134 & -0.286 & 0.554 \\
\hline IL- $6^{5}$ & 190 & 2.035 & 0.007 & -0.355 & -0.958 & 0.248 & 0.248 & 0.106 & -0.388 & 0.601 & 0.674 & -0.813 & -1.281 & -0.346 \\
\hline IL-10 5 & 191 & 1.854 & 0.22 & -0.588 & -1.167 & -0.010 & 0.046 & 0.086 & -0.389 & 0.561 & 0.723 & -0.177 & -0.622 & 0.268 \\
\hline IL-17 6 & 144 & 26.162 & $<0.001$ & -0.395 & -1.083 & 0.293 & 0.260 & 1.375 & 0.951 & 1.798 & $<0.001$ & $-1.110^{*}$ & -1.716 & -0.504 \\
\hline TNF- $a^{6}$ & 190 & 1.396 & $\mathrm{NA}^{7}$ & 1.000 & 0.490 & 1.511 & $<0.001$ & 0.440 & 0.021 & 0.860 & 0.039 & 0.014 & -0.382 & 0.410 \\
\hline$N / L^{8,5}$ & 144 & 0.573 & 0.009 & 0.163 & -0.266 & 0.592 & 0.456 & 0.217 & -0.095 & 0.5303 & 0.173 & 0.499 & 0.212 & 0.787 \\
\hline $\mathrm{IL}-2 / \mathrm{IL}-10^{5}$ & 144 & 0.928 & 0.077 & 0.450 & -0.027 & 0.926 & 0.064 & 0.219 & -0.161 & 0.598 & 0.259 & -0.150 & -0.526 & -0.226 \\
\hline IL-6/IL-10 5 & 190 & 1.687 & $<0.001$ & 1.141 & 0.586 & 1.696 & $<0.001$ & -0.685 & -1.141 & -0.230 & 0.003 & -1.011 & -1.442 & -0.580 \\
\hline IL-17/IL-10 5 & 144 & 1.368 & 0.001 & -0.028 & -0.595 & 0.539 & 0.922 & 0.318 & -0.133 & 0.769 & 0.167 & -0.761 & -1.209 & -0.314 \\
\hline
\end{tabular}

Note:

1. Adjustment for age, gender, marital status, BMI, education, and severity of depressive symptoms

2. Taking the healthy control group as reference, if the $\beta$ is positive, the level of inflammatory marker is higher than that of healthy control group, and if the $\beta$ i negative, the level of inflammatory marker is lower than that of healthy control group

3. Bold font denotes $p<0.05 / 15=0.0033$

4. According to the scatter plot and statistical test, these data are approximately normally distributed, so the linear model is used in modeling;

5. According to the scatter plot and statistical test, these data are skewed distributed, but approximately normally distributed after log conversion, so gamma link model is chosen for modeling.

6. The data dispersion is too high to be modeled when linear or gamma with log link mode is used, so tweedie with log link mode is used instead.

7. Unable to compute the initial model log likelihood due to numerical problems

8. N/L: Granulocyte/lymphocyte ratio

\section{Discussion}

We investigated the relationship between serum levels of inflammatory markers and symptomatic severity of bipolar disorder among 228 drug-naïve patients with bipolar disorder. Our study has three major findings. First, in BDD, both pro-inflammatory markers and anti-inflammatory markers are lower than those in $\mathrm{HC}$ in spite of the symptomatic severity. Second, in BDM, the serum levels of pro-inflammatory markers first elevate then decrease with the worsening of the manic symptoms. Third, the imbalance of pro-inflammatory markers and anti-inflammatory markers is seen in both BDD and BDM, which is shown by an alteration (decrease in BDD and increase in BDM) in the IL-6/IL-10 ratio when the symptoms are mild and a decrease in the IL-17/IL-10 ratio when the symptoms are severe.

Contrary to the view from studies in major depressive episode (MDD) [22] [23]that serum levels of both pro-inflammatory cytokines and ant-inflammatory cytokines slightly increase in depressive episode, our study finds that both pro-inflammatory cytokines and anti-inflammatory cytokines decrease in bipolar depression. In addition, the decrease of the ratios of IL-6/IL-10 and IL-17/IL-10 found in this study also suggests that immune-inhibition is prominent in bipolar depression, especially in severe BD. Different from studies in MDD, few studies have ever assessed the relationship between the serum levels of inflammatory markers and depressive symptoms from studies in BDD. Several studies with small sample size have found that bipolar depression is associated with a proinflammatory state $[24,25]$. However, in two review studies $[26,27]$ which meta-analyzed the cytokines alteration in BD, no consistent conclusion about the relationship between cytokines and bipolar depression was reached since few studies on bipolar depression were included and the sample sizes of the 
included studies were all small. In addition, the heterogeneity between studies also played a part in this inconsistency[27]. A recent comparison study[28] base on a machine learning approach found that compared to healthy control, BDD had higher levels of C-C Motif Chemokine Ligand (CCL)3, CCL4, CCL5, CCL11, CCL25, CCL27, CXCL11, IL-9 and TNF-a. However, in this study, all the participants were medicated, potential confounders including age, gender, BMI, and tobacco or alcohol use were not controlled. In addition, the average age of the participants were bigger than those in our study (46.59 $\pm 10.8 \mathrm{VS}$. $27.8 \pm 10.7$ ), which might be another factor that makes their conclusion different from ours. In addition, the foregoing conclusion could not be duplicated by other studies either. For example, a study [29]from German showed that both pro-inflammatory and anti-inflammatory markers, but not CRP were inversely correlated with the severity and symptoms of major depression. A recent study [30]from China found that the serum levels of IL-13 and TNF-awere significantly lower in BDD than in MDD, and the serum levels of IL-4 and TNF-aincreased in the treatment response subgroup of BDD. In recent years, anti-inflammatory agents were expected to be a promising treatment target of MDD [31]and BDD[32]. However, negative results from large clinical trials [33, 34]not only overturns the conclusion from small studies that the adjunctive use of anti-inflammatory drugs might help improve depressive symptoms in MDD or BDD, but also make us rethink the relationship between serum levels of inflammatory cytokines and depressive symptoms. Moreover, pro-inflammatory cytokines are not always neurotoxic. Instead, low-dose of pro-inflammatory cytokines such as IL-6, TNF-a, and IFN-y have been proved to have a neuroprotective role[35].

The relationship between serum levels of inflammatory markers and BDM has been widely studied[36]. Most of previous studies find that both proinflammatory cytokines (IL-1RA[37], IL-1, II-2[38], sIL-2R[39, 40], IL-6[38, 41], sIL-6R[39], TNF-a[41], CXCL10[42], CXCL11[42], CRP[39], IL-17[43], IFN-Y[25] and IL18[44]) and anti-inflammatory markers (IL-4[38] and IL-10[45]) elevate in the manic episode. However, our study only partially supports this view. In our study, the elevation of pro-inflammatory markers was seen only in mild and moderate BDM, while in severe BDM the serum levels of pro-inflammatory were found to decrease. This finding is consistent with our previous hypothesis about the dynamic change of inflammatory markers over the course of a multi-system inflammatory disease. As far as we know, this has rarely been explored before. Although serum levels of some inflammatory cytokines like slL-2R[40], IL17[43], neural cell adhesion molecule 1(NCAM-1)[45], carcinoembryonic antigen (CEA)[46] and IFN-y[25] have been reported to be positively correlated with severity of manic symptoms, the relationship between serum levels of inflammatory markers, according to our study, seems to be more complicated than a positive correlation.

Inflammatory ratios except N/L, which are less affected by exercise, BMI, and other confounding factors than other commonly used markers of inflammation[47], have been rarely studied in BD before. Partially in line with previous reports [48]that N/L ratio is higher in BDM than in BDD or MDD, our study finds that $\mathrm{N} / \mathrm{L}$ ratio is significantly higher in severe BDM than in $\mathrm{HC}$, suggesting an imbalance in favor of innate immunity [49]. In addition, we also find that the ratios of IL-6/IL-10 and IL-17/IL-10 vary with the clinical phase of BD and severity of symptoms: in BDD, the IL-6/IL-10 ratio first decrease in mild BDD and then the IL-17/IL-10 ratio decrease in severe BDD; while in BDM, the IL-6/IL-10 ratio first elevate in mild BDM and then decrease with the IL-17/IL-10 ratio in severe BDM. That is to say, the IL-6/IL-10 imbalance is an early immune response to BD, while IL-17/IL-10 imbalance is an indicator of deterioration of BD. To our knowledge, these have not been reported before. Although the pathophysiological meaning of IL-6/IL-10 and IL-17/IL-10 balance is far from being interpreted clearly, it is possible to suppose that they play a role in homing of inflammatory cells and therefore in the outcome of inflammation[50].

This study has several strengths. First of all, it comprises of one of the largest sample of BD patients ever examined. Second, all the subjects were Han Chinese and drug-naïve, most of them were young, and active physical diseases and recent substance abuse were excluded, which provided us a good sample with great homogeneity. Third, generalized linear model was used to statistically analyze the data, whereas as many potential confounding factors as possible were adjusted for, so the power of test greatly improved. Fourth, as many inflammatory cytokines as possible were measured at the same time, which makes the assessment of the immune state of the subjects more multidimensional. Finally, all the blood samples were treated in the same way and in the same laboratory, thus minimized the effect of measurement deviation. However, several limitations should be addressed when interpreting the foregoing conclusion. First, its cross-sectional design limits causal conclusion about the relationship between serum levels of inflammatory markers and BD. Second, symptoms of $\mathrm{BD}$ were treated as a binary variable-depressive or manic symptoms, while other domains of symptoms like psychotic symptoms, or cognitive symptoms were not concerned, which had been proved to be associated with the levels of inflammation. Third, although we have the largest sample of BD ever examined, the sample might be still not big enough to detect some small but clinically meaningful effect of inflammatory marker on the symptomatic severity of BD, especially when the sample is divided into several subgroups. Fourth, some but fortunately not much inflammatory markers' data was too dispersed to build a good generalized linear model, therefore caution should be exercised when interpretating the corresponding results.

\section{Conclusion}

The serum level of inflammatory markers and the balance between pro-inflammatory markers and anti-inflammatory markers not only vary with the types of affective episode but also with the severity of affective symptoms. In BDD, immune-inhibition is persistent predominant, while in BDM, immune system is first activated then inhibited with the worsening of the symptoms. The dynamic change of serum inflammatory markers might not only help stage BD but also guide the future immune treatment of BD.

\section{Declarations}

\section{Acknowledgements}

The authors would like to gratefully acknowledge the contributions of all of the nurses, technicians, and patients that participated in this study. We especially thank professor Wen Chen from the School of Public Health, Sun Yat-sen University for her constructive suggestions on the statistical analysis in this study.

\section{Ethics approval and consent to participate}

This study were reviewed and approved by the Clinical Research Ethics Committee of the Third Affiliated Hospital of Sun Yat-Sen University. The research had been performed in accordance with the Declaration of Helsinki. Written informed consents had been attained from all the participants based on the principle 
of self determination.

\section{Consent for publication}

Not applicable.

Availability of data and materials

All data generated or analysed during this study are included in this published article and and its supplementary information file.

Competing interests

All authors report no biomedical financial interests or potential conflicts of interest.

Funding

Funding for this study was provided by Hospital Nursing Research Fund of the Third Affiliated Hospital of Sun Yat-Sen University (201805), third Affiliated Hospital of Sun Yat-Sen University, Clinical Research Program and the Natural Science Foundation of Guangdong Province, China (2018A030313489). The above mentioned funding bodies had no further role in the study design, collection, analysis and interpretation of data; in the writing of the report; and in the decision to submit the paper for publication.

Authors' contributions

Zhaoyu Gan and Nianhong Guan conceived and designed the study and Revised it for intellectual content. Zhaoyu Gan, Xiuhua Wu, Yingtao Liao, Zhihua Yang and Nianhong Guan took recruitment and management of the case. Zhongcheng Chen was in charge of biochemical measurement. Xiuhua Wu Zhongcheng Chen and Xiaolin Liang contributed to data analysis and interpretation. Xiuhua Wu and Zhongcheng Chen Drafted the Article. All authors read and approved the final manuscript.

\section{References}

1. Trede K, Salvatore P, Baethge C, Gerhard A, Maggini C, Baldessarini R: Manic-depressive illness: evolution in Kraepelin's Textbook, 1883-1926. Harvard review of psychiatry 2005, 13(3):155-178.

2. Kapczinski F, Magalhães P, Balanzá-Martinez V, Dias V, Frangou S, Gama C, Gonzalez-Pinto A, Grande I, Ha K, Kauer-Sant'Anna M et al: Staging systems in bipolar disorder: an International Society for Bipolar Disorders Task Force Report. Acta psychiatrica Scandinavica 2014, 130(5):354-363.

3. Castano-Ramirez OM, Sepulveda-Arias JC, Duica K, Diaz Zuluaga AM, Vargas C, Lopez-Jaramillo C: Inflammatory Markers in the Staging of Bipolar Disorder. A Systematic Review of the Literature. Rev Colomb Psiquiatr 2018, 47(2):119-128.

4. Rosenblat JD, McIntyre RS: Bipolar Disorder and Immune Dysfunction: Epidemiological Findings, Proposed Pathophysiology and Clinical Implications. Brain Sci 2017, 7(11).

5. Leboyer M, Soreca I, Scott J, Frye M, Henry C, Tamouza R, Kupfer DJ: Can bipolar disorder be viewed as a multi-system inflammatory disease? J Affect Disord 2012, 141(1):1-10.

6. Rosenblat JD: Targeting the immune system in the treatment of bipolar disorder. Psychopharmacology (Berl) 2019, 236(10):2909-2921.

7. Berk M, Conus P, Lucas N, Hallam K, Malhi GS, Dodd S, Yatham LN, Yung A, McGorry P: Setting the stage: from prodrome to treatment resistance in bipolar disorder. Bipolar Disord 2007, 9(7):671-678.

8. Kohler-Forsberg O, Sylvia L, Deckersbach T, Ostacher MJ, Mclnnis M, losifescu D, Bowden C, McElroy S, Calabrese J, Thase M et al: Clinically relevant and simple immune system measure is related to symptom burden in bipolar disorder. Acta Neuropsychiatr 2018, 30(5):297-305.

9. Queissner R, Pilz R, Dalkner N, Birner A, Bengesser SA, Platzer M, Fellendorf FT, Kainzbauer N, Herzog-Eberhard S, Hamm C et al: The relationship between inflammatory state and quantity of affective episodes in bipolar disorder. Psychoneuroendocrinology 2018, 90:61-67.

10. Lassmann H: Targets of therapy in progressive MS. Mult Scler 2017, 23(12):1593-1599.

11. Gonzalez-Quintela A, Alende R, Gude F, Campos J, Rey J, Meijide LM, Fernandez-Merino C, Vidal C: Serum levels of immunoglobulins (IgG, IgA, IgM) in a general adult population and their relationship with alcohol consumption, smoking and common metabolic abnormalities. Clin Exp Immuno/ 2008 , 151(1):42-50.

12. Osimo EF, Cardinal RN, Jones PB, Khandaker GM: Prevalence and correlates of low-grade systemic inflammation in adult psychiatric inpatients: An electronic health record-based study. Psychoneuroendocrinology 2018, 91:226-234.

13. Elliot AJ, Heffner KL, Mooney CJ, Moynihan JA, Chapman BP: Social Relationships and Inflammatory Markers in the MIDUS Cohort: The Role of Age and Gender Differences. J Aging Health 2018, 30(6):904-923.

14. Gonzalez-Gil EM, Cadenas-Sanchez C, Santabarbara J, Bueno-Lozano G, Iglesia I, Gonzalez-Gross M, Molnar D, Gottrand F, De Henauw S, Kafatos A et al: Inflammation in metabolically healthy and metabolically abnormal adolescents: The HELENA study. Nutr Metab Cardiovasc Dis 2018, 28(1):77-83.

15. Lojko D, Rybakowski JK: Atypical depression: current perspectives. Neuropsychiatr Dis Treat 2017, 13:2447-2456.

16. Akcan U, Karabulut S, Ismail Kucukali C, Cakir S, Tuzun E: Bipolar disorder patients display reduced serum complement levels and elevated peripheral blood complement expression levels. Acta Neuropsychiatr 2018, 30(2):70-78. 
17. Stapel B, Sieve I, Falk CS, Bleich S, Hilfiker-Kleiner D, Kahl KG: Second generation atypical antipsychotics olanzapine and aripiprazole reduce expression and secretion of inflammatory cytokines in human immune cells. J Psychiatr Res 2018, 105:95-102.

18. Frey BN, Andreazza AC, Houenou J, Jamain S, Goldstein BI, Frye MA, Leboyer M, Berk M, Malhi GS, Lopez-Jaramillo C et al: Biomarkers in bipolar disorder: a positional paper from the Intemational Society for Bipolar Disorders Biomarkers Task Force. Aust N Z J Psychiatry 2013, 47(4):321-332.

19. Hamilton M: A RATING SCALE FOR DEPRESSION. Journal of Neurology, Neurosurgery \&amp; Psychiatry 1960, 23(1):56-62.

20. Young RC, Biggs JT, Ziegler VE, Meyer DA: A Rating Scale for Mania: Reliability, Validity and Sensitivity. British Journal of Psychiatry 2018, 133(5):429435.

21. Yatham LN, Kennedy SH, Parikh SV, Schaffer A, Bond DJ, Frey BN, Sharma V, Goldstein BI, Rej S, Beaulieu S et al: Canadian Network for Mood and Anxiety Treatments (CANMAT) and International Society for Bipolar Disorders (ISBD) 2018 guidelines for the management of patients with bipolar disorder. Bipolar Disord 2018, 20(2):97-170.

22. Dahl J, Ormstad H, Aass HC, Malt UF, Bendz LT, Sandvik L, Brundin L, Andreassen OA: The plasma levels of various cytokines are increased during ongoing depression and are reduced to normal levels after recovery. Psychoneuroendocrinology 2014, 45:77-86.

23. Young JJ, Bruno D, Pomara N: A review of the relationship between proinflammatory cytokines and major depressive disorder. J Affect Disord 2014, 169:15-20.

24. Poletti S, Mazza MG, Calesella F, Vai B, Lorenzi C, Manfredi E, Colombo C, Zanardi R, Benedetti F: Circulating inflammatory markers impact cognitive functions in bipolar depression. $J$ Psychiatr Res 2021, 140:110-116.

25. Remlinger-Molenda A, Wojciak P, Michalak M, Rybakowski J: [Activity of selected cytokines in bipolar patients during manic and depressive episodes]. Psychiatr Pol 2012, 46(4):599-611.

26. Munkholm K, Brauner JV, Kessing LV, Vinberg M: Cytokines in bipolar disorder vs. healthy control subjects: a systematic review and meta-analysis. $J$ Psychiatr Res 2013, 47(9):1119-1133.

27. Modabbernia A, Taslimi S, Brietzke E, Ashrafi M: Cytokine alterations in bipolar disorder: a meta-analysis of $\mathbf{3 0}$ studies. Biol Psychiatry 2013, 74(1):15-25.

28. Poletti S, Vai B, Mazza MG, Zanardi R, Lorenzi C, Calesella F, Cazzetta S, Branchi I, Colombo C, Furlan R et al: A peripheral inflammatory signature discriminates bipolar from unipolar depression: A machine learning approach. Prog Neuropsychopharmacol Biol Psychiatry 2021, 105:110136.

29. Schmidt FM, Schroder T, Kirkby KC, Sander C, Suslow T, Holdt LM, Teupser D, Hegerl U, Himmerich H: Pro- and anti-inflammatory cytokines, but not CRP, are inversely correlated with severity and symptoms of major depression. Psychiatry Res 2016, 239:85-91.

30. Mao R, Zhang C, Chen J, Zhao G, Zhou R, Wang F, Xu J, Yang T, Su Y, Huang J et al: Different levels of pro- and anti-inflammatory cytokines in patients with unipolar and bipolar depression. J Affect Disord 2018, 237:65-72.

31. Kohler O, Krogh J, Mors O, Benros ME: Inflammation in Depression and the Potential for Anti-Inflammatory Treatment. Curr Neuropharmacol 2016, 14(7):732-742.

32. Rosenblat JD, Kakar R, Berk M, Kessing LV, Vinberg M, Baune BT, Mansur RB, Brietzke E, Goldstein BI, McIntyre RS: Anti-inflammatory agents in the treatment of bipolar depression: a systematic review and meta-analysis. Bipolar Disord 2016, 18(2):89-101.

33. Gallagher PJ, Castro V, Fava M, Weilburg JB, Murphy SN, Gainer VS, Churchill SE, Kohane IS, losifescu DV, Smoller JW et al: Antidepressant response in patients with major depression exposed to NSAIDs: a pharmacovigilance study. Am J Psychiatry 2012, 169(10):1065-1072.

34. Husain MI, Chaudhry IB, Khoso AB, Husain MO, Hodsoll J, Ansari MA, Naqvi HA, Minhas FA, Carvalho AF, Meyer JH et al: Minocycline and celecoxib as adjunctive treatments for bipolar depression: a multicentre, factorial design randomised controlled trial. Lancet Psychiatry 2020, 7(6):515-527.

35. Carlson NG, Wieggel WA, Chen J, Bacchi A, Rogers SW, Gahring LC: Inflammatory cytokines IL-1 alpha, IL-1 beta, IL-6, and TNF-alpha impart neuroprotection to an excitotoxin through distinct pathways. $J$ Immunol 1999, 163(7):3963-3968.

36. Rosenblat JD, Mclntyre RS: Bipolar Disorder and Inflammation. Psychiatr Clin North Am 2016, 39(1):125-137.

37. Liu HC, Yang YY, Chou YM, Chen KP, Shen WW, Leu SJ: Immunologic variables in acute mania of bipolar disorder. J Neuroimmuno/ 2004, 150(1-2):116122.

38. Brietzke E, Stertz L, Fernandes BS, Kauer-Sant'anna M, Mascarenhas M, Escosteguy Vargas A, Chies JA, Kapczinski F: Comparison of cytokine levels in depressed, manic and euthymic patients with bipolar disorder. J Affect Disord 2009, 116(3):214-217.

39. Bai YM, Su TP, Tsai SJ, Wen-Fei C, Li CT, Pei-Chi T, Mu-Hong C: Comparison of inflammatory cytokine levels among type I/type II and manic/hypomanic/euthymic/depressive states of bipolar disorder. J Affect Disord 2014, 166:187-192.

40. Tsai SY, Yang YY, Kuo CJ, Chen CC, Leu SJ: Effects of symptomatic severity on elevation of plasma soluble interleukin-2 receptor in bipolar mania. $J$ Affect Disord 2001, 64(2-3):185-193.

41. Kim YK, Jung HG, Myint AM, Kim H, Park SH: Imbalance between pro-inflammatory and anti-inflammatory cytokines in bipolar disorder. J Affect Disord 2007, 104(1-3):91-95.

42. Barbosa IG, Rocha NP, Bauer ME, de Miranda AS, Huguet RB, Reis HJ, Zunszain PA, Horowitz MA, Pariante CM, Teixeira AL: Chemokines in bipolar disorder. trait or state? Eur Arch Psychiatry Clin Neurosci 2013, 263(2):159-165.

43. Li HZ, Hong W, Wang ZW, Yuan CM, Li ZZ, Huang J, Zhang C, Li NN, Lin ZG, Fang YR: [Correlation between Expression of Peripheral IL-17 Protein and Aggression of Bipolar Mania]. Fa Yi Xue Za Zhi 2016, 32(1):40-44.

44. Munkholm K, Weikop P, Kessing LV, Vinberg M: Elevated levels of IL-6 and IL-18 in manic and hypomanic states in rapid cycling bipolar disorder patients. Brain Behav Immun 2015, 43:205-213. 
45. Jesudas BR, Nandeesha H, Menon V, Allimuthu P: Relationship of elevated neural cell adhesion molecule 1 with interleukin-10 and disease severity in bipolar disorder. Asian J Psychiatr 2020, 47:101849.

46. Bulut M, Cati S, Gunes M, Kaya MC, Kaplan I, Ozkan M: Evaluation of serum inflammatory markers in treatment-resistant manic patients and adequate responder manic patients. Psychiatry Res 2019, 272:73-79.

47. Gibson PH, Croal BL, Cuthbertson BH, Small GR, Ifezulike Al, Gibson G, Jeffrey RR, Buchan KG, El-Shafei H, Hillis GS: Preoperative neutrophil-lymphocyte ratio and outcome from coronary artery bypass grafting. Am Heart J 2007, 154(5):995-1002.

48. Fusar-Poli L, Natale A, Amerio A, Cimpoesu P, Grimaldi Filioli P, Aguglia E, Amore M, Serafini G, Aguglia A: Neutrophil-to-Lymphocyte, Platelet-toLymphocyte and Monocyte-to-Lymphocyte Ratio in Bipolar Disorder. Brain Sci 2021, 11(1).

49. Mazza MG, Tringali AGM, Rossetti A, Botti RE, Clerici M: Cross-sectional study of neutrophil-lymphocyte, platelet-lymphocyte and monocyte-lymphocyte ratios in mood disorders. Gen Hosp Psychiatry 2019, 58:7-12.

50. Tampoia M, Abbracciavento L, Morrone M, Fumarulo R: IL-6/IL-10 Ratio as A Prognostic and Predictive Marker of the Severity of Inherited Epidermolysis Bullosa. Iran J Immunol 2017, 14(4):340-349.

\section{Supplementary Files}

This is a list of supplementary files associated with this preprint. Click to download.

- rawdata.xls 